\title{
The Integrated Mission Design Center (IMDC) At NASA Goddard Space Flight Center
}

\author{
G. Karpati, J. Martin, and M. Steiner \\ NASA Goddard Space Flight Center \\ Greenbelt MD, 20771 \\ 301-286-4468 \\ http://imdc.gsfc.nasa.gov/ \\ K. Reinhardt \\ Air Force Research Laboratory \\ Kirtland AFB, NM 87117 \\ 301-286-8411 \\ Kitt.C.Reinhardt.1@gsfc.nasa.gov
}

\begin{abstract}
NASA Goddard has used its Integrated Mission Design Center (IMDC) to perform more than 150 mission concept studies. The IMDC performs rapid development of highlevel, end-to-end mission concepts, typically in just 4 days. The approach to the studies varies, depending on whether the proposed mission is near-future using existing technology, mid-future using new technology being actively developed, or far-future using technology which may not yet be clearly defined. The emphasis and level of detail developed during any particular study depends on which timeframe (near-, mid-, or far-future) is involved and the specific needs of the study client. The most effective mission studies are those where mission capabilities required and emerging technology developments can synergistically work together; thus both enhancing mission capabilities and providing impetus for ongoing technology development.
\end{abstract}

\section{TABLe of Contents}

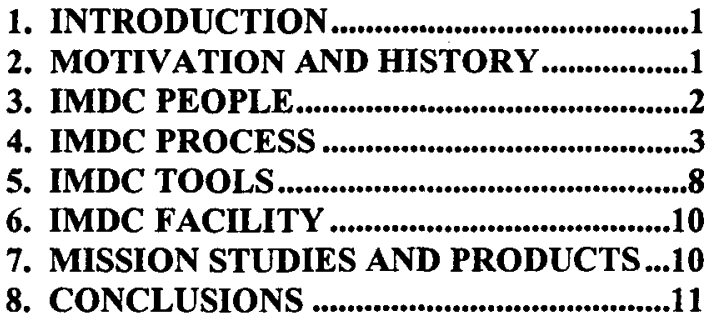

\section{INTRODUCTION}

The Goddard Space Flight Center (GSFC) has developed a comprehensive environment for concept development and engineering study of remote sensing instruments and aerospace missions. Called the Integrated Design Capability (IDC), the environment is actually comprised of two closely related study centers: the Instrument Synthesis and Analysis Laboratory (ISAL) and the Integrated Mission Design Center (IMDC). Studies performed span a broad range of remote-sensing and in-situ measurements conducted from aircraft and balloon platforms, Shuttle or Space Station based endeavors, as well as free-flying satellites. The ISAL provides a flexible, high fidelity capability for the design, modeling and analysis of remote sensing and in-situ instruments. The IMDC provides end-to-end mission studies, encompassing the full life cycle of both flight and ground elements of these missions. The first study was performed in the IMDC in June 1997. Since then, more than 150 mission studies have been accomplished. The IMDC part of the IDC is the focus of this paper.

Early IMDC studies were structured to be performed for clients who had a fairly well developed concept of an instrument or instrument suite needed for a specific scientific investigation. They sought assistance from the IMDC in finalizing approaches for orbits, spacecraft bus performance, data return scheme, operations concept, and life-cycle cost. Studies typically focused on a single satellite at a time, usually in low Earth orbit. To support these studies, a systematic set of modeling and analysis procedures were established resulting in mission concept study products that were able to meet client needs and requirements. More recent IMDC studies have entailed concepts for multiple satellites having significantly different characteristics e.g., mother ship with "children" satellites or two or more different satellites flying in formation to form a distributed system in space.

\section{MOTIVATION AND HISTORY}

The motivation for establishing the IMDC at the Goddard Space Flight Center (GSFC) arose in the mid 1990's during a period of shrinking NASA resources (money and labor) and the movement towards greater efficiency. The

\footnotetext{
${ }^{1}$ U.S. Government work not protected by U.S. copyright

${ }^{2}$ IEEEAC paper \#1175, Updated December 9, 2002
} 
perception within GSFC at the time was that the traditional mission design process was inefficient, it required too many meetings, over-taxed the ever-decreasing resource pool, took too long to complete, and yielded inconsistent results that did not always meet expectations.

Prior to establishing the IMDC, the GSFC approach for conducting space mission design studies consisted of assigning a team of engineers the task of analyzing and/or developing a desired mission concept. The study team members had specialized expertise pertinent to the particular mission scenario being studied, and in some cases had previously worked together. However, the team members participated in the study only on a part-time basis. Although the teams met periodically as needed to coordinate and discuss design problems, the team members independently conducted the majority of the analysis and design work in their home offices. Consequently, progress was often very slow due to the lack of focus, coordination, and interplay among the different discipline engineers, as well as to the normal workload demands on each team member. Also, the study client was not necessarily involved in the total design process, significantly reducing the probability of the design being fully satisfactory. It became increasingly evident to GSFC mission planners that a more flexible and integrated mission design capability was needed to meet client needs.

In early 1996 a coordinated effort was undertaken at GSFC to create a comprehensive space mission design center focused on increased integration, flexibility, fidelity, timeliness, and cost-effectiveness. Paramount goals in this effort were to significantly reduce the time required to analyze and develop space mission concepts, while also increasing usefulness for the study client. The approach implemented by GSFC was modeled after the principles of collaborative and concurrent design engineering, where study clients would closely collaborate with the facility's resident team of discipline engineers throughout the space mission study period.

When the initial plans for the IMDC were formulated, the decision was made to build on past mission study successes, while focusing on the recognized problems of coordination and integration. Two important features were added to the new IMDC process that were absent from the old traditional approach. The first feature was the development of an integrated design center laboratory - a designated physical space - to facilitate the mission design studies. The design center laboratory would house a collection of workstations and servers capable of sharing information during the design process. The permanent office space would provide the study team a central location to gather and develop the mission concepts in a focused, undisturbed environment. The second new feature involved the nature of the workforce that would conduct the studies. The engineers assigned to the IMDC would remain part of their home organization, and would continue to have other work assignments, but once an IMDC study was initiated, the team members would work virtually full-time on the study through completion.

Overall, the IMDC process enabled a mission concept study that had previously taken three months or more to be completed in less than one week. This accelerated schedule allowed increased client participation throughout the process. The paradigm used by GSFC and the synergistic relation between the elements of the design center is graphically illustrated in Figure 1.

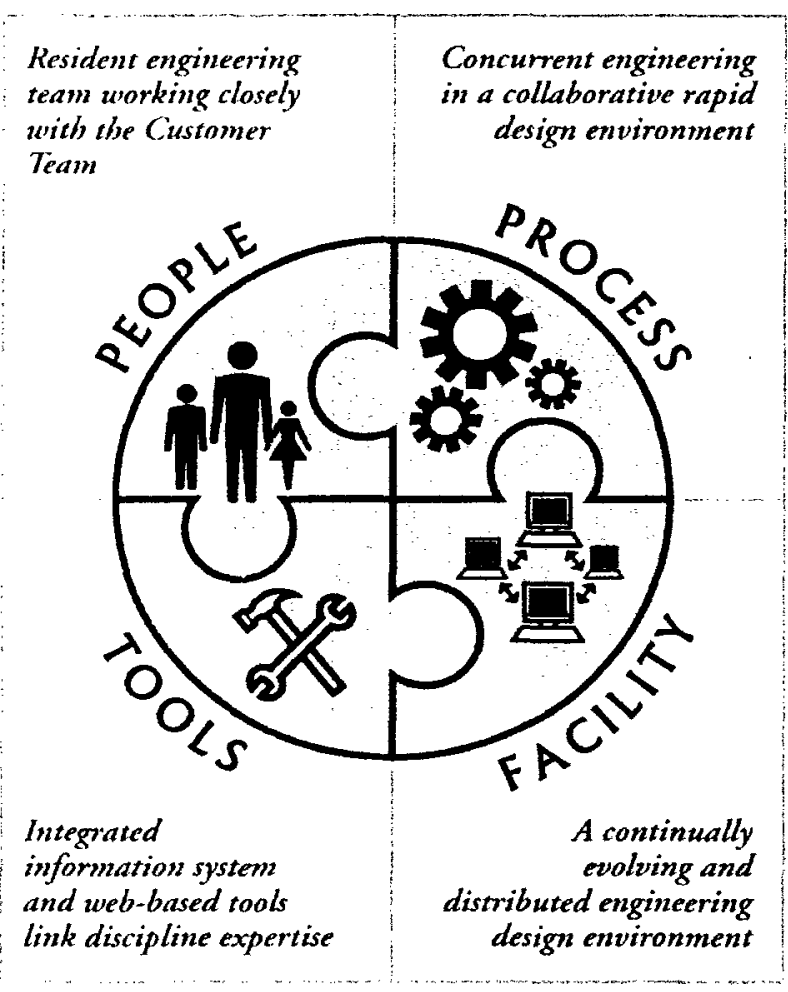

Figure 1: Design Center Paradigm

The four elements of the IMDC structure, people, process, tools and facility, constitute the themes of the discussion that follows.

\section{IMDC PEOPLE}

The cornerstone of the IMDC interactive design process is people - the team of expert GSFC Discipline Engineers working in concert with the client (or client team). The Discipline Engineering Team (DET) is selected based on team member space flight mission experience, either flight article or supporting ground system, and the ability to work in a collaborative, concurrent environment. Most DET members have 10-15 years of subsystem design experience with space flight missions.

As mentioned above, the IMDC Discipline Engineers would be drawn from their "home" organizations, but would not be removed from those organizations. At the discretion of their home organization, the DET members are either assigned to 
the IMDC for a specific time, either full-time or on a parttime basis. The assignment emphasizes currency of engineering knowledge for each discipline. Remaining part of the discipline's home organization makes it easier for an individual discipline engineer to call on others in the home organization for information or other help needed for a study.

This approach also benefits the home organization since nobody is "lost" to that organization and the IMDC affords an excellent opportunity for each discipline engineer to be exposed to all aspects of a mission, thus producing a broadened perspective which the individual will take to future work.

Using the intended science investigation and the instrument(s) needed to conduct that investigation as a starting point, a typical IMDC study covers essentially every other aspect of space mission conceptual design. Science team planning, final scientific analysis of the returned data, publication of scientific results, and related items are the only ones excluded. To support the covered areas, the DET currently includes the disciplines of: 1) flight dynamics and attitude control; 2) propulsion and propellant; 3 ) command and data handling; 4) communications systems and RF links; 5) flight software; 6) solar array, battery, and power electronics; 7) mechanical and structures; 8) thermal control; 9) mission operations and ground systems; 10) launch vehicle capability; 11) reliability and safety; 12) integration and testing; 13) mission cost estimation; 14) mission risk analysis; 15) orbital debris and deorbit analysis; 16) orbit environment assessment; and 17) risk management. A Team Lead and a Systems Engineer guide the DET through each design session.

\section{IMDC PROCESS}

During the earliest IMDC studies, it was clear the key to achieving a successful mission study was to satisfy client expectations for concept study product timeliness, maturity, and fidelity. Hence, the first challenge for each study was to establish the reasonable set of client expectations to be met. The next was balancing the study requirements with IMDC study resources, in terms of analysis tools and available labor. Further, in some cases it also became clear that for studies to be successful it was necessary to identify the 'key' or especially challenging mission requirements prior to starting the IMDC study, allowing team members to adequately prepare, gathering supporting information or performing preliminary analysis of characteristics or parameters that would be critical to successful completion of the study.

\section{Methodology}

The systematic process for performing studies has been organized into three phases: Preparation, Execution, and Wrap-up. Although the fundamental study methods for all three phases have remained essentially unchanged for the later studies, several details of early software tools and methods of have required modification over the years.

The Preparation phase is started when a client completes an on-line 'IDC Request for Support' form, which identifies general information concerning the mission type, scope of study requested, and time frame the support is needed. Following staff review of this request, a Pre-work meeting is scheduled with the client, and an IDC secure server account is established enabling the client to complete a webbased Pre-work questionnaire. The questionnaire has roughly 100 entries, and is designed to capture detailed information on the science and mission objectives, instrument concepts, orbit parameters, pointing requirements, mission operational concept, and desired study trades and products. Often, more than one Pre-work meeting is held between the client and IMDC staff prior to and after completion of the questionnaire to discuss study concerns and support issues. Whenever possible, we start the Preparation phase 2 to 4 months before the actual study period.

In the Execution phase, also referred to as the study phase, the full study is conducted by the DET, led by the Team Leader and System Engineer. Depending on the study scope, this phase typically takes $4-5$ days. A typical 4-day IMDC study execution flow, or timetable, is shown in Figure 2. The timetable provides a guide for the sequence of activities followed by the IMDC team throughout the study.

\section{Day 1}

AM Client briefing to IMDC team on mission and science objectives, and IMDC objectives

- IMDC systems engineer briefs DET on prework results and engineering approach

PM Coordination meeting with full MMDC DET and client team to review current baseline concepts, identify open issues, and schedule open splinter sessions

- Client collaboration and mission design process

\section{Day 2 \& 3}

AM Coordination meeting with IMDC and client teams, mission design process continues

PM Coordination meeting with full IMDC DET and client team to review current baseline concepts, identify open issues, and schedule open splinter sessions

- Client collaboration and mission design process

\section{Day 4}

AM - IMDC DET completes final analysis, reviews final end-to-end conceptual design, prepares final presentation package for delivery to client

PM Final design study results presented to client team

- Action items resulting from client briefings are reviewed and are dispositioned

- Short debriefing held with client

- DET begins close-out of action items and finalizes documentation

Figure 2: Typical 4-day IMDC Study Execution Flow. 


\section{Concurrent and Collaborative Engineering Process:}

The term, concurrent in the IMDC environment conveys a three-part meaning: 1) all phases of a mission lifecycle are considered at the same time, 2) all disciplines work in conjunction with each other, making tradeoffs for the benefit of the overall system, and 3) as the study progresses, any needed changes to requirements or overall conditions are taken into account by all affected Discipline Engineers. This produces converging on a conclusion that is consistent across all aspects of the mission design. The IMDC is a collaborative study environment where the Discipline Engineers, System Engineer, Team Leader and client interact in a tightly coupled iterative process. During the study, every subsystem communicates with virtually all other subsystems. The free-form interaction between subsystems is shown graphically in Figure 3, with the shaded box representing any subsystem interacting as needed with other subsystems.

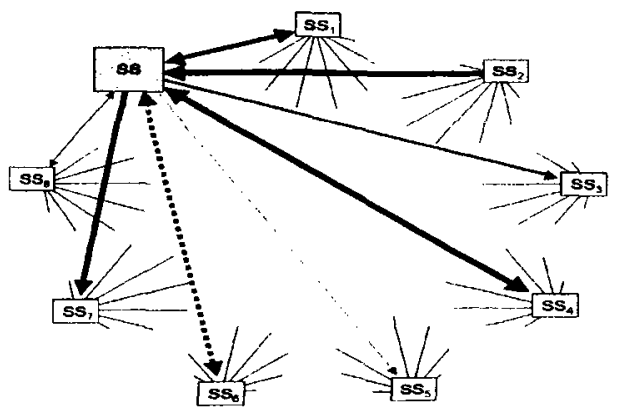

Figure 3: Inter-discipline interaction

The level of interaction between individual Discipline Engineers is dependent upon the mission requirements and complexity of the task. The information flow can be formal or informal, and can take various forms from verbal through electronic, and can proceed in one direction or both. The content of the communication and the strength of interdependence vary greatly as a function of the coupling between the disciplines for the particular parameter involved. The level of interaction for a specific parameter can also change from one study to another. This multichannel information exchange serves as the main forum of concurrent analysis during the study.

The success of the IMDC study process depends on the diligent and knowledgeable contribution of the individual subsystem engineers. To ensure this process consistently occurs; a set of procedures has been established. Each DET member reports a mandatory set of key engineering parameters to the Study Lead Systems Engineer on a periodic basis throughout the study. Figure 4 illustrates how the system concept is built up by collaborative synthesis with contributions from all subsystems.

The DET is required to submit a first estimate of key design parameters at the onset of the study. Thereafter, in a roll-call manner during 'tag-up' meetings twice a day, each

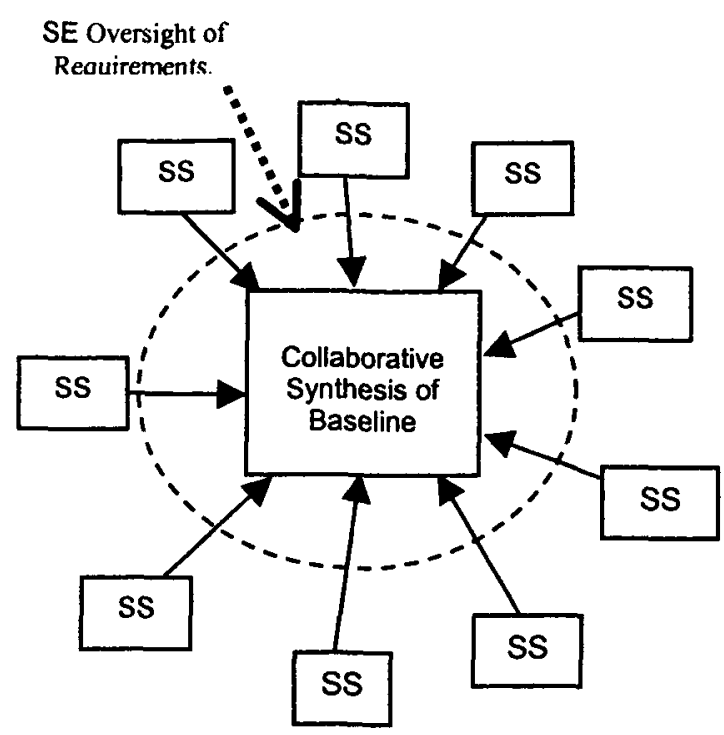

Figure 4: Key Parameters Reporting

subsystem engineer presents and defends his latest set of subsystem design parameters and explains the underlying design to both the client and the entire IMDC team. This iterative and interactive approach provides an informal peer review process for validation of the subsystem and system designs through discussions, while allowing effective disseminates of relevant subsystem data and design issues to the client and IMDC team.

Throughout the mission design process, the Systems Engineer and Team Leader maintain a database of requirements, some of which have been subdivided and allocated to the affected disciplines to use in their detailed work. During the coordination meetings, problems with meeting these allocated requirements are discussed and the allocations are changed if necessary. In Figure 4, this function was represented with the dotted circle entitled "SE Oversight of Requirements", while Figure 5 (next page) illustrates the same function from the vantage point of the overall allocation and management of requirements.

The following outlines the basic flow of the IMDC study process throughout the study period. The overall view, illustrating the iterations described below is depicted in Figure 6 (next page).

1) The initial requirements and parameters that address the most challenging mission requirements are assessed through concurrent analysis by the IMDC team, who at the same time also identifies key system dependencies. This interdiscipline interaction is represented by section 1-A, part of Iteration 1 within Figure 6 . This activity is a simplified representation of what is depicted in Figure 3.

2) All DET members work together to collaboratively synthesize a credible straw man concept. Concept synthesis starts with input data from mission requirements and initial assumptions for power, mass, propellant and data budgets, 


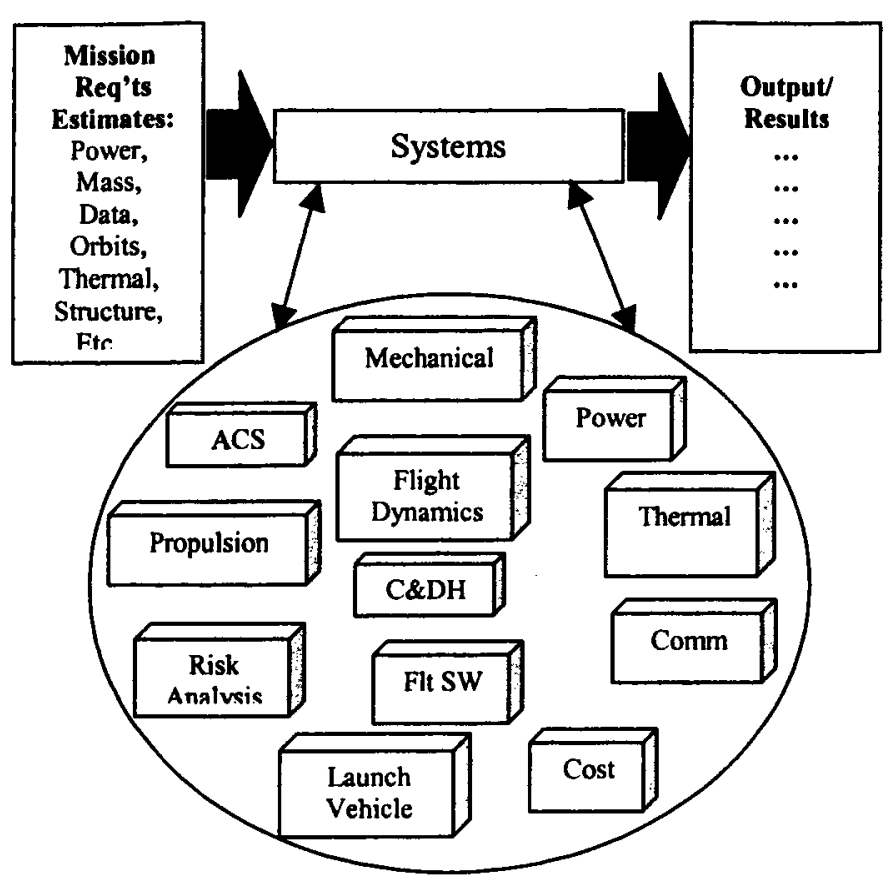

Figure 5: Requirements Management

orbit requirements, and any previous analysis results for the instrument (e.g., from the ISAL) and spacecraft. This collaboration and key parameters reporting is represented by section 1-B, also part of Iteration 1 within Figure 6. This activity is a simplified representation of what is depicted in Figure 4. The product of this effort, the straw man concept, is represented by section 1-C, the conclusion of Iteration 1 within Figure 6.

3) Using the straw man concept as the starting point, the baseline concept is gradually developed with subsystem and system dependencies and circum-dependencies gradually incorporated in an iterative series of concurrent analysis and the collaborative synthesis integrated under systems management oversight. Sections 2-A and 2-B within Iteration 2 are very similar to the concurrent analysis and collaborative synthesis performed in Iteration 1, but performed with more mature information. The product of this effort, 2-C, is the baseline concept.

4) This process of concurrent analysis and collaborative synthesis is repeated as necessary until the inter- and circum-dependent parameters converge into a coherent final mission concept baseline design. This is shown as the Iteration 3 part of Figure 6. Some studies may require more or less than 3 iterations, but the 3 iterations shown in Figure 6 are typical. This process concludes when the final baseline design provides sufficient information to allow development of credible performance and cost models with contingencies

\section{The Original IMDC Process and its Limitations:}

The initial IMDC process was developed to deal with a relatively simple set of mission concept requirements, such as a single spacecraft in low Earth orbit. It also assumed that all studies could be performed with essentially the same timeline. With the success of the early studies, demands and expectations grew, and more ambitious studies were requested having significantly more complex requirements.

Some clients requested the IMDC to explore the ramifications of very large performance trades and options, such as considering the liabilities and benefits of using nextgeneration and generation-after-next technologies on mission performance and capability; some requested

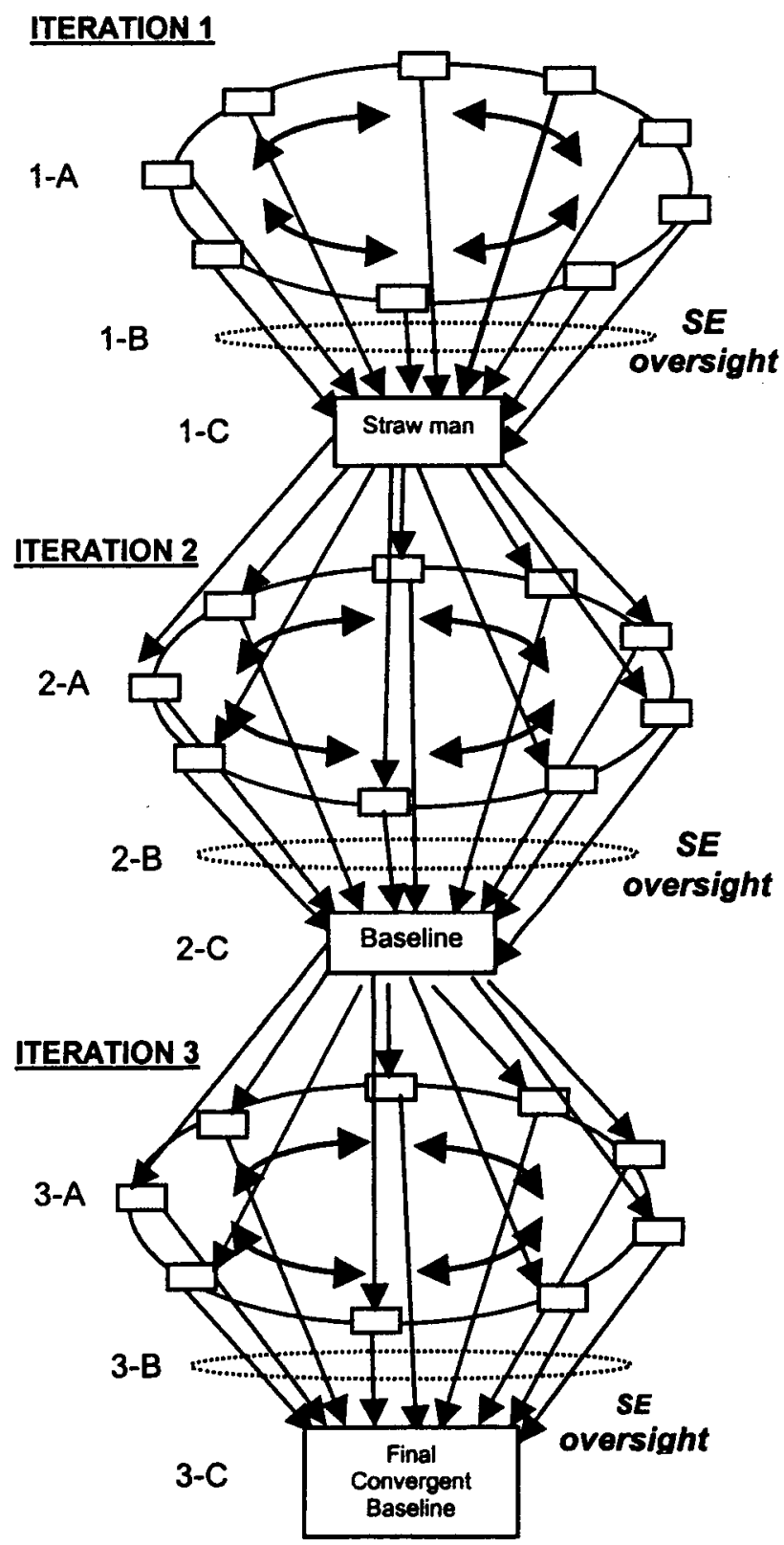

Figure 6: Iterative Process Flow, Overall View 
analyses of complex formation flying missions with fleets of dozens or even hundreds of spacecraft; some requested analyses of several very different spacecraft in a single mission (e.g., free flyer satellites with deployer and mother ships). One of the challenges presented with the multiple spacecraft missions was that the IMDC 'data exchange platform' was only able to accommodate a single spacecraft.

Another important aspect of the increased complexity was the need for more design iterations. Since engineering is fundamentally an iterative process, as complexity grew, so did the need for iterations. At first, the IMDC attempted to complete the more complex and demanding concept designs by intuitively applying its basic original processes. As one would expect, frequent analysis deficiencies and 'roadblocks' popped up. Generally, when problems arose, the existing processes were uniquely modified and adapted, mostly in ad hoc fashion, to putout the 'fires' and allow the study to continue.

\section{Improved Processes:}

Although most early high complexity studies were completed to the client's satisfaction, success was due more to the efforts of the well-oiled team than to the rigor of the processes employed. It became clear that the processes on hand had severe limitations, and significant improvements were necessary to satisfy the higher complexities. A concerted effort was undertaken to do that. It was recognized that one of the keys to achieve the desired improvements is to adapt to the greater variety of needs. The new mission studies requested were not only more complex than before, they were more varied. As an ever-increasing variety of dissimilar missions followed one another in the IMDC, it was understood that a generic set of design processes could not provide the necessary operations improvements. Effective improvement would only be achieved by developing distinctly different processes for the different study types.

\section{Study Types and Process Types:}

The studies requested by the IMDC clients could be grouped into four basic types; 1) Proposal effort in response to a NASA Announcement of Opportunity (AO), 2) Early mission formulation, 3) Advanced concepts for future missions, and 4) Special studies or system architecture trades.

Unique processes were developed to support these four study types. The following paragraphs provide a description of the key features of each study type, and highlights of the specific characteristics of the custom tailored processes developed for them.

\section{Mission Design for a Proposal Effort:}

Typically this type of study is performed to aid a team responding to a NASA AO. The client requesting it sometimes already has a team with several experts skilled in various disciplines. Some teams know what they want to do scientifically, but have not yet defined the spacecraft bus, ground system or mission operations concept. Other teams may have a fairly mature concept of the mission with welldefined requirements, well beyond just the definition of the payload. In either case, the IMDC must produce a specific design with a mature operations concept, generally including an end-to-end design and associated cost estimate.

\section{Processes Used to Support Mission Design for a Proposal Effort:}

This process emphasizes precision in design, and accuracy in costing, playing to the traditional strengths of the IMDC. Accordingly, other than enhancing and improving the costing services as described below, only minimal amounts of custom tailoring and deviation from the original IMDC processes and timelines was required.

Progress towards completion is usually predictable and straightforward. The focus is on the technical and engineering correctness of the baseline configuration. The Team Leader allows ample time for extensive technical discussions during tag-up meetings, since the exchange of ideas serves these study types particularly well as an informal peer review, possibly preempting Red Team concerns. The IMDC has extensive experience with missions preparing to submit a bid. In addition to the technical work, the IMDC team is usually cognizant of existing AO's, and is well suited to offer the PI and/or proposal leader advice on preparing the final proposal.

The other main concern is the accuracy of the Cost Report. The IMDC has significantly improved the grassroots costing tables and aligned them with the standard Goddard Work Breakdown Structure. Even more importantly, an independent Cost Analyst has been added to the facility. In that position, a parametric costing expert, using Price-H (a parametric cost estimating tool), generates an independent parametric cost estimate for the spacecraft. In the final presentation, the grassroots and Price- $\mathrm{H}$ cost numbers are compared and assessed.

\section{Mission Design for Early Mission Formulation:}

The clients requesting this type of study are typically NASA earth and space scientists. The definition of the mission payload varies, from the "feasibility validated" state, up to relatively mature designs, and even to the use of hardware that has already flown. Beyond this, generally not much else of the mission design has been considered before coming to the IMDC. The contribution expected from the IMDC is one of two basic types, requiring very different approaches by the IMDC. These two sub-categories: validate the feasibility of a concept and explore and trade multiple options, along with the corresponding processes for each are outlined below under $a$ ) and $b$ ). 
Processes Used to Support Mission Design for Early Mission Formulation:

The emphasis in early mission formulation is on knowledge. These studies aim to put the very considerable collective knowledge of the IMDC team to use in formulating or consolidating the client's typically loose initial plans into a credible mission concept. The focus is on accuracy and professionalism while trying to find an optimal balance between today's technology and the possibilities of tomorrow.

a) Validate the Feasibility of a Concept:

Typically, the objective of this type of study is to develop mission requirements that could significantly affect the payload design, with study results primarily used to refine payload requirements. This type of study usually includes a rough order of magnitude cost estimate.

\section{Processes Used to Validate Feasibility of a}

\section{Concept:}

Orbit work can be critical for these studies; the shape of the spacecraft or even the feasibility of the mission may hinge on its accuracy. The IMDC has encountered clients who were unaware of the complexities of orbital dynamics for their mission, and formulated prework requirements or mission goals for unattainable or sub-optimal orbits. In some cases, the consequences of this threatened the success of the whole study. Unfortunately, orbit work is usually very time intensive. For a complex orbit, the work required to even enable an IMDC study can be of massive proportions. To overcome this, the IMDC has included an orbital dynamics representative as a standard member of the prework team. This person's task is to scout for and initiate orbital analysis prior to study start.

b) Explore and Trade Multiple Options:

This type of study refines a mission concept by completing a "puzzle" approach down-selection from the available building blocks (which can be a set of instruments, orbits, or other features). The IMDC is required to document the trades, and analyze effects and ramifications of those trades (sometimes with combinations and permutations) on end-to-end parameters, performance metrics, liftoff mass, or mission cost.

\section{Processes Used to Explore and Trade Multiple Options:}

The clients for these studies usually place less emphasis on a point design baseline. Instead they want such questions answered as "What if I do this?" or "What if I do that?" Accordingly, the original IMDC timeline is almost completely irrelevant for these studies. These are among the more difficult studies for the IMDC, calling for interpersonal management skills of the IMDC leadership working with the DET and study client. Heavy demands are placed on the facility's IT tool set as well. The tools must be flexible and modifiable to accommodate many design options and trades. In this case, possibly more than others, a negative finding may be as valuable as a positive one to the client.

This type of study presents a tough challenge in managing the IMDC team's time and effort; allocating and focusing the team's time and energy to the right areas, recognizing dead ends in time to avoid driving the design into an engineering culde-sac, and finally making sure the gears of the study click together (i.e., one subsystem may be waiting input from another for Option A, but that other subsystem is now working Option B).

The other side of the table may also test the interpersonal skills of the IMDC leadership. The client may feel that the IMDC session is a unique opportunity to have all their questions answered, and may call for an excessive workload. The IMDC leadership must at times down-negotiate the client's requests. This down-negotiation can occur in the middle of a study, but preferably should be done much before, during Prework negotiations. The IMDC experience has shown that three, or sometimes four distinctly different main options (or the number of distinctly different spacecraft types) are an upper limit that still allows the study to stay focused.

\section{Mission Design for Future Missions with Advanced Concepts:}

This client is typically a visionary scientist or technologist with a very advanced science observation concept that is far beyond the present state-of-the-art. The role of the IMDC is to provide an initial feasibility evaluation and technology needs assessment. That is usually accomplished by developing a straw man mission design, accompanied by citations of the technology developments required to make the mission feasible. Cost estimates are required but less important, as their inherent uncertainty is recognized.

\section{Processes Used to Support Mission Design for Future Missions with Advanced Concepts:}

For these missions the emphasis usually is on technology. In some cases, an assistant to the SE is designated as the cognizant focal point for the technologies used in the mission. He/she researches the technologies in advance, presents them to the team at the opening of the session, and is present during the study for consultation and monitoring. In addition, the IMDC may request the hands- on support of GSFC Technologists. 


\section{Special Studies or System Architecture Trades:}

This client already has a network or a functioning team staffed with high-level skilled experts and a clear concept of the mission to be accomplished. For this type of study, the IMDC provides metrics used in high-level roadmap formulation, compares pros and cons of different possible system configurations, or similar activities which require end-to-end mission analysis from the technical viewpoints represented by the IMDC disciplines. The emphasis is on consistency and realism of the IMDC product.

\section{Processes Used to Support Special Studies or System Architecture Trades:}

This work often consists of a series of IMDC runs wherein each run can almost be regarded as a study in itself. The individual study session focus can be any of the previous three types described above, and may even change from individual study to individual study within the same series of IMDC runs for the same client.

What truly sets such a study series apart from other work is the extreme need for consistency between different parts of the study. To insure the desired degree of consistency, a part of the study time is dedicated to review and compare work done to that point. At both the system and subsystem levels, checks are performed for the credibility, evenness and consistency of the approach, the engineering, the techniques and technologies, and the published values. The product of such a session can be comparison and trending charts, which the client may use as parametric design tools.

While the four basic mission study types generally differ in analysis approach and products required, the same system of collaborative and concurrent study is used. The implementation of the collaborative and concurrent development approach has proven to be very successful and a significant value.

\section{IMDC TOOLS}

Even though the above four basic mission study types generally differ in analysis approach and products required, many of the same tools are used. Having the best tools is crucial in enabling the IMDC to meet client expectations. The tools the IMDC uses fall under two general categories:

- Infrastructure tools such as the data exchange platform used to support concurrent engineering. These tools are typically unique, either developed in house for use by the IMDC team, or developed in or for vary similar design centers.

- Discipline tools that are provided by the discipline engineers and their home organizations. They can be Government-Off-The-Shelf (GOTS), Commercial-OffThe-Shelf (COTS), or custom made. Frequently they are developed in house, in many instances by the discipline engineers themselves for personal use.

\section{Tools Management}

The operational concept for IMDC has been to encourage the DET to use tools with which they are already familiar, and which have received the endorsement of their home organization. Rather than prescribing a set of centrally approved and managed tools, the DET is allowed to use any of their own tools as they see fit. This "grass roots" approach has proven successful. On one hand, with a centrally managed tool set every new DET member would require training and a learning period before being able to use the tools in the IMDC, increasing cost and complicating staffing. On the other hand, when engineers are allowed to use the tools from their home engineering organization, they become instantly productive after joining the IMDC, and the results are more readily accepted by the home organizations. Lastly, with central management the tools inventory of the IMDC could easily become rigid. Conversely, in a grassroots environment evolution is continuous and spontaneous. We believe the wide variety of tools presently used in the facility is one of the main keys of the success of the IMDC.

Due to the close collaboration between engineers in the IMDC, there is intense cross-pollination of tool knowledge between members of the same discipline, and even between engineers of different disciplines. This is natural: when an engineer sees a colleague use a superior tool, he/she will become interested in exploring it as well. This has an invigorating effect that carries beyond the IMDC, back to the engineer's home organization. Additionally, the client sometimes retains certain tools used in the IMDC for mission design for use in the subsequent phases of their program. When an IMDC tool is carried over into the project that results from these studies, one of the IMDC goals is fulfilled: to provide products that can be used in the entire life cycle of projects.

In a similar manner, the IMDC serves as a catalyst and low risk proving ground for new tool development. New tools are often developed in the IMDC or are tried and validated there. One technique is to run a new tool in parallel with the existing one to verify output, catch bugs, errors, and improve modeling. This practice also has benefits that are felt far beyond the IMDC.

Lastly, IMDC management and the IT support team are always evaluating commercial tools and developing new ones to support and control the study process. The IMDC is constantly on the lookout for any tool that can further enhance the process of rapid design.

\section{Tools to support concurrent engineering}

The tools to directly support concurrent engineering (such as the electronic data interface platform), are perhaps the most challenging tools in use in the IMDC. These tools keep the IMDC team coordinated throughout the design. They are are under the direct management of the System Engineer, assisted by a team of IT support personnel. 
After an initial period where data exchange was mostly accomplished via traditional means (paper, email), a sophisticated data exchange platform, the IMDC System for Information Sharing (ISIS) was developed and put in use. ISIS was coded in html, to be accessed via a web browser (such as Netscape or Internet Explorer). ISIS algorithms were derived from experience with early studies, thus ISIS was perfectly adapted for those relatively simpler studies: one single spacecraft in low Earth orbit, with no configuration variations, no options to explore, and with relatively consistent study timelines. For missions that fit the above profile, ISIS was superb. Besides serving as a data exchange platform, it also had the following advanced features: It automatically populated the subsystem requirements fields on the subsystem pages with requirements derived from the Prework Questionnaire; It served as a requirements management aid in the interaction between System Engineer and DET; and it automatically tallied up resource estimates for mass, power and grassroots cost.

As demands for studies with more options grew, the limitations of ISIS became first prominent, then painful. Greatest among these limitations was the cast iron rigidity of ISIS -- inflexibility inherent in any html application. No line items, let alone new configurations of additional spacecraft, could be added or modified without involving the system developer - a process that could not be accomplished in near real time during a study. By the time a particular change got implemented, other fixes were urgently needed. That promised an endless backlog of ISIS improvements. Apparently the imagination and creativity of the study clients outran one's ability to foresee all twists needed to be implemented in ISIS.

The IMDC gave up the attempt to build a rigid system that would be so accommodating that it could serve the more complicated studies. Instead, it was recognized that the emphasis must be on flexibility: the ability to modify many aspects of data transfer and display in near real time to adapt it to even the most unusual study need.

A new system level design tool and information exchange platform dubbed EXcel Information eXchange (EXIX) was developed to supersede the polished, but less flexible ISIS. To allow virtually unlimited flexibility, EXIX was coded in Visual Basic to run under a system of inter-accessible but separate Excel applications. Adapting the lessons learned from ISIS, EXIX was slowly matured over a year of handson operations in several studies in the IMDC.

After EXIX's operational algorithm had been thoroughly field tested, polished, and fine-tuned during the IMDC field trials, IDC professional IT specialists were commissioned to re-code the same algorithm (amplified with numerous enhancements) into a standalone (not Excel) data exchange platform application. The new tool will be put into operational use by early CY2003. By consolidating the data exchange functions into a professional standalone application, the following goals are achieved which would not be possible for EXIX: provide integral connections to design databases, provide hooks for future automatic report generation, and allow for future links into other subsystems.

\section{DET tools}

The discipline engineers have access to a wide mix of COTS, GOTS, and homegrown tools. For example, in the area of trajectory design, the IMDC engineer can support studies covering the range from balloon flights to low earth orbit, geosynchronous orbits, libration orbits, and on into deep space. The mission can consist of a single spacecraft, constellations of spacecraft, or spacecraft in a formation flying architecture. The IMDC engineer can evaluate communication link performance within any of the frequency bands allocated for space use, determining expected data quality while accounting for extra attenuation due to atmosphere and rainfall for any location on earth. The following is a partial list of tools currently used by the Flight Dynamics, Power, Mechanical, Communication, and Cost Analysis Discipline Engineers in the IMDC.

Flight Dynamics tools used:

- Satellite Tool Kit

- SWINGBY

- GTDS

- GMAN

- MAnE

- Custom Target Acquisition Tool

- Freeflyer Engineer

- Solar Cycle Modeling Tools

- Mathlab

- Mathematica

Power Subsystem tools used:

- Electronic Power Spacecraft Simulation Tool

- Solar Power Modeling Tools

- Orbit Dynamics Energy Balance Tool

- Battery Sizing Tool

- Voltage Trade Sheet

- Radiator Degradation Tool

Mechanical / Structural tools used:

- Ideas

- Pro-E

- Autocad

- Pastran / Nastran

- On-Line Launch Vehicle Selection Tools

RF Communications tool used:

- CLASS

Parametric Cost Analysis tool used:

- PRICE-H

All subsystem engineers perform maintenance, improvement, and upgrading, of their tools, with little or no 
central coordination from the IMDC. Plans are in place, however, to better assist the subsystem engineers by evolving the system level data platform. In its next version, the platform will extend beyond the interface boundaries of the subsystems, and will reach into the subsystem inner areas, to automate the transfer of information. That will relieve the DET from periodic reporting (now done manually on the electronic platform), helping to resolve the problems of timeliness and discipline in reporting. The baseline will always be up-to-date, as the reported numbers will be automatically drawn from the evolving subsystem designs. Additionally, the new system will insure correctness and consistency in accounting; no line item will be overlooked.

\section{IMDC FACILITY}

The IMDC facility plays a critical role in the successful completion of IMDC studies, by providing a dedicated and convenient place for the collocation of the key players, equipment, information, and communication capabilities. The laboratory houses a state of the art collection of software, computer workstations and servers capable of sharing information seamlessly during the design process. A layout of the IMDC Facility, including the laboratory, conference room, facilities support, as well as supplemental seating and support is shown in Figure 7.

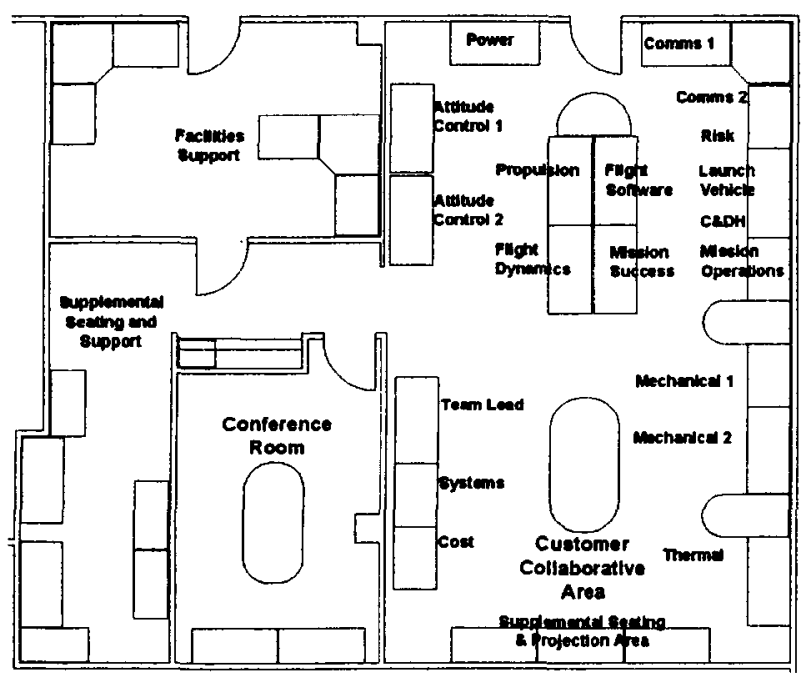

Figure 7: IMDC Facility Layout

The laboratory section of the IMDC occupies nearly 1000 square feet, and contains roughly 20 work areas, each having a designated engineering workstation. A main objective of the laboratory layout is to promote and enhance inter-discipline communications. On one hand electronics equipment and software in the room are all implemented to maximize every interaction. On the other hand the room itself also is designed to enhance client and study team interaction. Team members sit at their workstations and converse with each other and the client while reviewing design data and related information on workstation monitors. Informal conversations occur in the lab as people move freely from one area to the next. The client is encouraged to maintain a presence in the room so the team can obtain quick feedback to questions and design issues.

The laboratory has a conference table centered in the front of the room that the clients occupy during the study. Three projection screens dominate the front wall, one of which can be raised to reveal an electronic whiteboard. The three projection screens are each served by a ceiling-mounted projector tied into an integrated audio-visual system. Each video image is controlled individually, allowing for the projection of the same image on all three screens, or for a unique feed to each screen. Data and information can be selected for display from any engineering workstation monitor within the laboratory, as well as video from an image capture device, or the internal GSFC cable TV network. The system also allows for an external video input. The sound system is fed by a video source or a wireless microphone used to enhance the presenter or study lead's voice. The client occupies the conference table that provides computer links to the projection system, Ethernet connections to the Internet, and a teleconference capability. This enables them to collaborate with the study team, while also allowing linkage to colleagues and facilities outside the IMDC laboratory.

A separate collocated conference room provides space for splinter meetings, prework sessions, and a convenient isolated area for client use during study execution. Additional collocated space is allocated for equipment such as FAX machine, printers, copiers, and shredders that are required for optimum operations but would be disruptive if housed within the engineering laboratory. To ensure optimum facility support and minimize disruptions to study execution, facility support personnel are also collocated within the design center complex.

\section{Mission Studies ANd Products}

The primary operational objective of the IMDC is to support the wide range of client needs for mission concept studies. The IMDC client base is mainly comprised of NASA earth and space scientist teams and project offices within the NASA enterprises. The IMDC also supports study clients from academia, industry and the other Federal Agencies. Some client teams also have included personnel from foreign countries. Since its inception in June 1997, the IMDC has completed 153 studies. Of these, 82 were Space Science studies, 56 were Earth Science studies, and 15 were various other studies. Approximately $20 \%$ of all the studies had a technology focus. These studies were conducted primarily for NASA/GSFC, but some studies were done for other NASA centers, other U.S. Government agencies, academia and commercial clients. More information can be found at the following Web sites: http://idconline.gsfc.nasa.gov/, http://isal.gsfc.nasa.gov/, or http:/imdc.gsfc.nasa.gov/. 


\section{Conclusions}

GSFC has produced an Integrated Design Capability, of which the IMDC is part, for enabling rapid and concurrent development of space mission and instrument designs. These designs have been used for a variety of purposes, including:

1) Providing assistance to teams responding to Announcements of Opportunity,

2) Assisting teams who are in the formulation phase of a directed mission,

3) Providing a "third party" assessment of an existing mission design, and

4) Assisting in the process of developing a roadmap for far future space missions.

The Integrated Design Capability has produced significant benefits for Goddard Space Flight Center, including:

1) Improved consistency and quality of early mission and instrument studies within an accelerated schedule and reduced resources, and

2) Processes and products continuously improving to meet greater demand, higher expectations and more complex mission design concepts.

Future plans will evaluate expanded capabilities in areas such as knowledge management, modeling and simulations, higher fidelity products, and supporting broader lifecycle studies.

Gabriel Karpati received his MSEE in 1979 from the Technical University of Budapest. At NASA since 1985, he has focused primarily on systems engineering for spacecraft and science instruments. Mr. Karpati has supported numerous missions, such as COBE, HST, GGS, and has been the Instrument Systems Engineer for GSFC science instruments on Cassini and SIRTF. Currently he is the System Engineer at the GSFC Integrated Mission Design Center.

John Martin received his $A B$ in Physics in 1955 from Wheaton College, Wheaton Ill. Joining NASA in 1958, he has focused primarily on systems engineering of ground systems and mission operations. Mr. Martin has supported numerous missions, such as Mercury, Apollo, ATS, TOMS-EP, and has been the Mission Operations Manager for the HST and Ground System Manager for Landsat-7.
Currently he is the Team Leader at the Integrated Mission Design Center of the Goddard Space Flight Center

Mark Steiner received his $B S$ in Electrical Engineering from Penn State in 1983 after which he joined NASA at the Goddard Space Flight Center. He has been a first-line supervisor and a Mission Manager for the Spartan Project Office for missions flown from the Space Shuttle in 1995 and 1996. He currently is a senior systems engineer, working

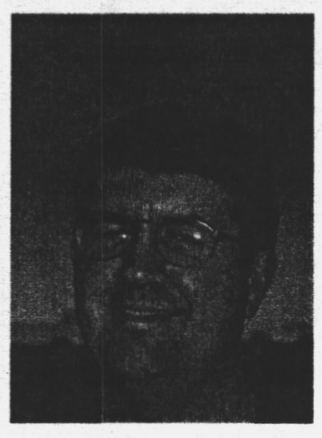
in the areas of advanced concepts and technology as well as providing mission systems engineering support for the Integrated Design Capability and the Earth Science and Space Science Directorates at Goddard.

Kitt Reinhardt received a $P h D$ in engineering physics from the Air Force Institute of Technology in 1994 and an MS in electrical engineering from the State University of New York in 1988. He is a member of the Air Force Research Laboratory (AFRL) Space Vehicles Directorate, where he recently served as Chief of the AFRL Space Power

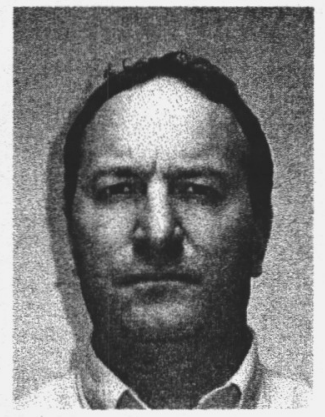
Generation Group. He is presently serving a 2-year detail at NASA's Goddard Space Flight Center where he is leading a concerted effort to establish research and development partnerships in the areas of advanced electronics, detectors, space power technologies, and space mission systems engineering 